\title{
Multiple Object Detection using GMM Technique and Tracking using Kalman Filter
}

\author{
Rohini Chavan \\ Research scholar \\ Bharati Vidyapeeth Deemed University College of \\ Engineering, Pune.
}

\author{
Sachin R. Gengaje \\ Research guide \\ Head Of Department \\ Electronics Dept. \\ WIT, Solapur.
}

\begin{abstract}
The continuous research in the technology of video acquisition devices increases the number of applications with best performance and less cost. For object recognition, navigation and surveillance systems, object detection and tracking are the indispensable steps. Object detection means segmentation of images between foreground and background objects. Object tracking establish the correspondence between the objects in successive frames of video sequence. In this paper, we have proposed algorithms consists of two stages i.e. object detection using Gaussian Mixture Model (GMM) and multiple moving objects tracking using Kalman filter. While tracking the moving object, problems occur during occlusion of persons with each other. However, it can be effectively deal with various video sequences such as indoor, outdoor and cluttered scenes. The experimental results shows that proposed algorithm achieve accurate, robust and efficient results for detection as well as for tracking the foreground objects from complex and dynamics scenes.
\end{abstract}

\section{General Terms}

Segmentation, Gaussian Mixture Model, Occlusion, Kalman Filter.

\section{Keywords}

Gaussian Mixture model, segmentation, Multiple object tracking, Kalman Filter, foreground object.

\section{INTRODUCTION}

The need to increase the installation of video surveillance system in public places significantly to protect from terrorist attack. There is a large significance of motion tracking in real time world as it enables various important applications such as to provide better security using video data in shopping malls and theatres, in medical therapy to improve the quality of life of patients, video abstraction to obtain automatic annotation of videos, traffic management to analyze traffic flow, video editing and to design futuristic video effects. Despite, video surveillance system is one of the most active research topic in computer vision. Mostly, every video surveillance system start with motion detection. Subsequent processes such as object tracking and behavior analysis are greatly dependent on it. Accurate detection of human motion can improve the performance of object classification as well as tracking.

The most challenging tasks for detection of moving objects are sudden change in an illumination of light, severe occlusion among the moving objects, shadows and non stationary background objects. A visual detection and tracking system required to generalize across large variations in object appearance such as posture, shape and facial expressions [1]. Background subtraction is the most commonly used method for moving object detection [2]. In this method construction of background modeling is the first step. It gives as a reference frame from first few frames of video sequences having only background objects. Image segmentation can be done by subtracting the background modeling frame from incoming frames of video sequences. Later, the frame difference is check with threshold value. If the difference is greater than threshold then it represents as a foreground pixel and if it is less then it belongs to background pixel. The threshold value is set by the user in such a way that errors should be minimized. In this way thresholding gives output in terms of binary frame. At last, morphological operations can be done to remove all unwanted random noise pixels.

After motion detection, the next important thing in surveillance system is generally to track the foreground objects from one frame to another in video sequence. It has been very critical job to apply in congested situations due to fault and less accuracy in segmentation of objects. For robust tracking, it is important to deal with shadows at motion detection and handle the occlusion both at segmentation level and at tracking level. According to survey on various tracking approaches, it can be classified as deterministic and probabilistic tracking [2]. For the former, Mean Shift (MS) [3] is the widely used technique because of its simplicity and effectiveness. It utilizes the parameters of data distribution for tracking multiple possible candidate. It is computationally very efficient but regular MS tracker loose the tracks due to fast changes occurred in the motion of the objects. However the quality of this algorithm performance degraded in case of severe occlusion occurred and similar kind of objects present in the video sequence.

On the other hand probabilistic tracking methods such as Particle filter and Kalman filter can model the important information such as position and velocity of tracked object in terms of probability distribution function and recursively updated with current measurements [4].

In this paper, we proposed Gaussian Mixture Model (GMM) technique for robust and accurate object detection and Kalman filter for efficient tracking of moving object in dynamic scene [2]. There are many other methods for moving object detection such as Background Subtraction, Inter frame differencing, Non parametric Kernel Density estimation and edge detection technique. Out of these techniques, Gaussian Mixture Model (GMM) is very efficient technique for motion detection. But it also has some kind of noise which overcome by morphological operations and gives accurate result.

The rest of the paper is organized as follows: A literature survey regarding different approaches for object detection and tracking are mention in section 2 . In section 3 , proposed techniques for detection and tracking of moving objects are presented. Section 4 gives experimental results for various indoor and outdoor datasets. Finally, we conclude the paper in section 5 . 


\section{RELATED WORK}

\subsection{Object detection}

Accurate motion detection in video surveillance system is crucial for different important application areas including abnormal event detection, person identification, gender classification and human gait characterization. The first step of the detection process is to detect the foreground object. It provides a classification of the pixels from video frames into either foreground or background pixels. Many researchers has given various approaches like background subtraction, optical flow and frame differencing method for detection of the objects.

Background subtraction algorithm (BS) [3] compute the absolute difference between the current frame and reference frame from video scene to get segmented image at the output. All the pixels associated with the same object can be connected to each other by performing a connected component analysis. All connected components are considered as foreground regions and with the help of morphological operations all unwanted pixels and small regions are eliminated.

Optical flow method [4] can compute the flow vectors of moving objects over time to detect foreground regions from video sequence. Even though the camera is moving still this technique can detect the moving objects. But it is computationally complex and used in real time applications for crowd analysis and dense motion with specialized hardware. Under the assumption of brightness constancy and special smoothness, Optical flow method is used to describe coherent motion of points or features between image frames. In frame differencing method [4], image segmentation can be done by taking the difference between two successive frames in video sequence. This is very simple method of moving object detection but at the cost of accuracy.

Stauffer and Grimson [3] proposed an approach based on Gaussian mixture model (GMM) used for background modeling. This technique can be used reliably when there is small change occurred in the illumination of light, repetitive motions from clutter and after a long time changes takes place in the scene. However, GMM suffers some problems in the process of object detection such as it consider entire frame as a foreground when suffering a sudden illumination change. In [5], new update algorithm for learning mixture model were presented. They also proposed a method for detecting moving shadows using an existing mixture model. Z. Zivkovic [6] presented Adaptive Gaussian Mixture Modeling technique in which the parameters of the component of the mixture can be constantly changed for each pixel. K. Toyama, J. Krumm [7], the mixture of Gaussian model was added with the concepts given by region level and frame level considerations and performance of the proposed algorithm is evaluated using Wallflower database whose ground truth for particular image is available.

Marko Heikkila and Matti Pietika [8] proposed a texturebased method for modeling the background and detecting moving objects using nonparametric approach. In this paper, a general nonparametric kernel density estimation technique was used for modeling a statistical representation of the scene background. The probability density function for each pixel intensity is evaluated using directly from the data instead of any parameter assumption. In [9] [10], Nonparametric kernal density estimation technique was proposed. In [11], The background model is estimated using pixel by pixel basis and samples at each pixel is clustered into the set of codeword. J.
Kato, T. Watanabe, S. Joga [12], adopted a Hidden Markov Model (HMM) approach for accurate estimation of background modeling. A. Monnet, A. Mittal [13], constructing the background from first few frames of video sequence by separating it into equal sized blocks and calculating an edge histogram for each block.

\subsection{Object tracking}

Moving object tracking is the process of locating the position of the target in time from video sequences. It approximate the path of multiple moving objects. Each of Kalman filter, Particle filter, Mean Shift tracking algorithm and Edge Detection plays an important role in computer vision. Tracking algorithm can be classified into two major groups such as state space approach like particle filter or Kalman Filter and kernel based approach like Mean Shift Algorithm [14 ].

The recursive filters such as Kalman and Particle filter are based on state space approach can estimate the state of the system using probability distribution function. These approaches are able to recover from lost tracks makes them most useful tracking algorithms [15]. Of course they may require high computational cost and more memory because of their recursive approach. The Mean Shift algorithm [16] is a nonparametric method. It is an iterative kernel based deterministic procedure which converges to a local maximum by finding the mean of the distribution by iterating in the direction of maximum increase in probability density. It is basically an iterative expectation maximization clustering algorithm executed within local search regions. The mean shift tracker provides accurate localization and it is computationally feasible. One of the most important disadvantage of this algorithm is that it does not work properly when the color of target candidate is similar to background and probably we may lose the important data. Of course there is another problem of mean shift technique when there is severe occlusion among the people. Hence Mean Shift cannot track the object in all frames.

Kalman filter algorithm [16] belongs to state space approach class of tracking algorithm. It can solve the tracking problems based on the state space equation and the measurement equation. The two steps for tracking the object using Kalman filter are prediction and correction. In prediction step, current time state estimate is measured from previous time step. This estimate is also known as a priori state estimate. It does not require any measurement values. Kalman filter estimates the position, velocity and acceleration of the object in each frame of sequence. It gives accurate result when there should not be any large variation in the speed of moving target. It can operate by taking linear model and Gaussian noise for better results with minimum mean square error [17]. It is a recursive estimator means that while estimating the current state it requires previous state and its current measurements. These two are sufficient to estimate the current state. Kalman filter averages the prediction of system state with new measurement by using weighted average phenomenon so as to get more accurate estimated values.

\section{PROPOSED METHODOLOGY}

This paper proposes a moving object detection algorithm which is based on Gaussian Mixture Model and object tracking using feature based Kalman filter algorithm to handle multiple objects. The system is fully automatic and requires no manual input of any kind for initialization of tracking. Kalman filter motion model is with features like centroid and area of moving objects in video scene using information 
obtained by detection to determine whether split or merge occurred. This technique achieves efficient tracking of multiple moving objects under the confusing situations.

\subsection{Object detection using GMM}

Gaussian Mixture Model (GMM) is basically one of the most popular technique to construct the background model for segmentation of moving objects from background. GMM technique assigns number of Gaussian distributions for each pixel to estimate reference frame. If there is no any variations in the pixel values then all Gaussian distributions approximate the same values. In that case only one distribution is exist and the other distributions are not important at all. However on other side, if pixel values are changes continuously then constant number of Gaussian is not always sufficient to estimate the background model. In that case it is necessary to determine approximate number of Gaussian.

A Gaussian Mixture Model(GMM) is a parametric probability density function. It is expressed in terms of weighted sum of Gaussian component densities. GMM is basically used as a parametric model which estimate the probability distribution function based on various object features. In computer vision technology, it is difficult to detect multiple moving objects in case of severe occlusion and dynamic scene changes occurred. In order to implement the background subtraction method to identify the moving objects from each portion of video frames, background modeling is always the first step. This background modeling can be done with the help of Gaussian Mixture Model. To get the desired result, all incoming frames from video sequence are subtracted from a reference background modeling frame and compare the difference with threshold value to segment the image between foreground and background. Finally, any other random pixels which are detected as a foreground pixels can be eliminated to improve the foreground mask.

\subsubsection{Algorithm of Gaussian Mixture Model}

In order to give a better understanding of the algorithm used for background subtraction, following steps were adopted to achieve the desired results:

Firstly, we compare each input pixels with the mean of the associated elements. If the value of a pixel is close enough to a chosen elements mean, then that element is considered as the matched element. In order to be a matched element, the difference between the pixels and mean must be less than the element's standard deviation which is scaled by factor $\mathrm{D}$ in the algorithm.

b) Secondly, update the Gaussian weight, mean and standard deviation (variance) to demonstrate the new obtained pixel value. For non matching elements, the weight 'w' decreases where as mean and standard deviation remains constant. It is dependent on learning rate ' $p$ ' in relation to how fast they change.

c) Thirdly, evaluate the elements which are part of the background model. For this a threshold value is applied to the element weight ' $w$ '.

d) Finally, identify the foreground pixels which are not match with any elements determined as a background.

\subsubsection{Formulas of GMM}

A Gaussian Mixture Model can be formulated as follow:

$P\left(x_{t}\right)=\sum_{i=1}^{k}\left(\omega_{\mathrm{i}, \mathrm{t}} n\left(X_{t} ; \mu_{i, t}, \sum i, t\right)\right.$

Where obviously,

$\sum_{i=1}^{k}\left(\omega_{\mathrm{i} t}\right)=1$
The mean of such a mixture equals

$\mu_{\mathrm{t}}=\sum_{\mathrm{i}=1}^{\mathrm{k}} \omega_{\mathrm{i}}, \mathrm{t} \mu_{\mathrm{i}, \mathrm{t}}$

that is, the weighted sum of the means of the component densities. Where $\mathrm{i}$ be the variable which represents the current pixel in frame, $\mathrm{K}$ is the number of Gaussian distributions, and $t$ represents time (i.e., the frame index), ${ }^{\omega}$ is an estimate of the weight of the ith Gaussian in the mixture at time $t, \mu$ is the mean value of the ith Gaussian in the mixture at time $t$, $\sum i, t$ is the covariance matrix of the ith Gaussian in the mixture at time $\mathrm{t}$

\subsection{Object tracking using Kalman filter}

Kalman Filter (KF) gives the optimal implementation of the Bayes estimator when the system to which it is applied is linear, and the dynamic system related noises are Gaussian with zero mean. This definition justifies the requirement of a more detailed analysis of the model to be used with the KF estimator[16].

Mathematically it is an evaluator which predict and update the states of wide range of linear processes. In Kalman filter, we consider a tracking system where $X_{k}$ is the state vector which represent the position, velocity and dynamic behavior of the object, where subscript $\mathrm{k}$ indicate the discrete time. The main aim is to estimate the value of $X_{k}$ from the measurement $Z_{k}$. Following are some mathematical description of Kalman filter which are divided into four phases-

\subsubsection{Process equation}

$x_{k}=A x_{k-1}+w_{k-1}$

Where A represent transition matrix and $\mathrm{X}_{\mathrm{k}}$ is the state at time $\mathrm{k}-1$ to $\mathrm{k}$. Vector $\mathrm{w}_{\mathrm{k}-1}$ is the Gaussian process noise $\mathrm{N}($.$) with$ the following normal probability distribution $\mathrm{p}(\mathrm{w})$.

$p(w) \sim N(O, Q)$

\subsubsection{Measurement equation}

$$
Z_{k}=H x_{k}+V_{k}
$$

Where $\mathrm{H}$ is the measurement matrix and $\mathrm{Z}_{\mathrm{k}}$ is the measurement observed at time $\mathrm{k}-1$ to $\mathrm{k}$ respectively. $\mathrm{V}_{\mathrm{k}}$ is the Gaussian measurement noise. N(.) with normal probability distribution $\mathrm{P}(\mathrm{v})$.

$p(v) \sim N(O, R)$

\subsubsection{Time update equation}

Equation (1) and (3) describe a linear model at time k. As $x_{k}$ is not measured directly, therefore the information provided by measured $Z_{k}$ is used to update the unknown states $\mathrm{x}_{\mathrm{k}}$ Apriori estimate of state $\widehat{x}_{\bar{k}}$ and covariance error $\widehat{x}_{k}$ estimate is obtained for the next time step $\mathrm{k}$

$$
\begin{aligned}
& x_{k}=A x_{k-1}+w_{k-1} \\
& P_{\bar{k}}=A P_{k-1} A^{T}+Q
\end{aligned}
$$

\subsubsection{Measurement update equation}

These equations are associated with the feedback of the system. The objective is to estimate posteriori estimating $\widehat{x}_{k}$ which is a linear combination of the priori estimate and the new measurement $Z_{k}$. These equations are given below 
$K_{k}=P_{\bar{k}} H^{T}\left(H P_{\bar{k}} H^{T}+R\right)^{-1}$

$\widehat{x}_{k}=\widehat{x}_{\bar{k}}+K_{k}\left(Z_{k}-H \widehat{x}_{\bar{k}}\right)$

$P_{k}=\left(1-K_{k} H\right) P_{\bar{k}}$

$\mathrm{K}$ is the Kalman gain which is computed by above the measurement update equations. After that aposterior state estimate $\hat{x}_{k}$ and aposterior error estimate $P_{k}$ is computed by the measurement $Z_{k}$. The time and measurement equations are calculated recursively with previous aposterior estimate to predict new aprior estimate. The recursive behavior of estimating the states is one of the highlights of the Kalman filter.

We choose centroid and size of tracking window as the feature value to describe moving object. After the moving object have been segmented, some process need to be required for tracking them. First to assign tracking window for each moving object in the scene. Tracking window size is slightly larger than image size. It not only reduce the noise interference but also reduce image processing time and increase the speed of operation.

Kalman filter tracking model can be divided into three submodules such as motion model, feature matching and model update.

\subsubsection{Motion estimation model}

Kalman filter used for tracking is defined in terms of its states, motion model and measurement equation matrix $X_{k}$ is an eight dimensional system state vector which can be represented as

$x_{k}=\left[x_{o, k}, y_{o, k}, l_{k}, h_{k}, v_{x, k}, v_{y, k}, v_{l, k}, v_{h, k}\right]^{T}$

where $x_{o, k}$ and $y_{o, k}$ represent horizontal and vertical centroid coordinate. $l_{k}$ and $h_{k}$ represent half width and half height of tracking window. $v_{x, k}, v_{y, k}, v_{l, k}$ and $v_{h, k}$ represent their speed respectively. After the state equation and measurement equation of motion model are defined in the next frame, Kalman filter can be used to estimate the object location.

\subsubsection{Feature matching}

Each moving object is described by its centriod and tracking window, the horizontal and vertical centroid coordinates and area of the $i^{\text {th }}$ object in the $\mathrm{k}^{\text {th }}$ frame were respectively described as $x_{k}^{i}, y_{k}^{i}$. First centroid distance function between the $\mathrm{i}^{\text {th }}$ object in the $\mathrm{k}^{\text {th }}$ frame and $\mathrm{j}^{\text {th }}$ object in the $\mathrm{k}+1^{\text {th }}$ frame is defined as

$$
D(i, j)=\frac{\left|\sqrt{\left(x_{k}^{i}-x_{k+1}^{j}\right)^{2}+\left(y_{k}^{i}-y_{k+1}^{j}\right)^{2}}\right|}{\max _{n}\left|\sqrt{\left(x_{k}^{i}-x_{k+1}^{n}\right)^{2}+\left(y_{k}^{i}-y_{k+1}^{n}\right)^{2}}\right|}
$$

\subsubsection{Model update}

When the value of the cost function is found to be minimum then the parameters of Kalman filter motion model is need to be updated and use them as a input in the next frame. Model can be updated repeatedly until the object is disappeared.

\section{EXPERIMENTAL RESULTS}

\subsection{Object detection using GMM}

In order to analyze the robustness and effectiveness of proposed method, different experiments under different conditions are demonstrated in our paper. Gaussian Mixture Model is used to extract the moving object from video scene. The performance of this algorithm can be evaluated using various dataset downloaded from internet. Wallflower is open source database [7] which can be used to evaluate the performance of GMM detection algorithm. It includes five set of image sequences covering possible critical situations in background with indoor and outdoor scenes. All sequences are of size 160x120 pixels, sampled at $4 \mathrm{~Hz}$. Dataset also includes hand drawn ground truth images and text file providing the description of video for all sequences. Ground truth image is the binary image representing foreground mask of the specific frame in the sequence. We tested our method with the help of this database and achieved the results. Table 1. summarizes all test sequences along with their ground truth.

Table 1. Wallflower dataset with test sequence and ground truth

\begin{tabular}{|c|c|c|c|}
\hline $\begin{array}{c}\text { Sr. } \\
\text { No. }\end{array}$ & Name & $\begin{array}{c}\text { Test } \\
\text { Sequence }\end{array}$ & Ground Truth \\
\hline 1 & $\begin{array}{c}\text { Moved } \\
\text { Object } \\
\text { (MO) }\end{array}$ & $\begin{array}{c}\text { Waving } \\
\text { Tree } \\
\text { (WT) }\end{array}$ & \\
\hline 2 & $\begin{array}{c}\text { Camouflage } \\
\text { (C) }\end{array}$ & \\
\hline 3 & $\begin{array}{c}\text { Foreground } \\
\text { Aperture } \\
\text { (FA) }\end{array}$ & \\
\hline 5 & $\begin{array}{c}\text { Bootstrap } \\
\text { (B) }\end{array}$ & \\
\hline
\end{tabular}

To check the sensitivity of our proposed algorithm, we have calculated performance parameter values for different setting of parameters[16]. Because of huge amount of different combinations, only one parameter is varied at a time. The measurement are made for several video sequences including indoor and outdoor scenes. After experimentation, the results are evaluated for proper setting of parameters across a wide range of values. The same observation was made for all the measured sequences. This property significantly eases the selection of parameter values. Furthermore, the experiments have shown that a good set of parameters for any type of sequences performs well.

GMM based detection technique is very sensitive to small changes in parameter values. The results are evaluated using various settings of parameters like learning rate $\alpha$ and threshold value $\mathrm{T}$ for each sequence. Appropriate setting of $\alpha$ and $\mathrm{T}$ decided based on lowest value of total error for each setting. Output foreground mask for each sequence with appropriate setting of parameters like learning rate $\alpha$ and threshold $\mathrm{T}$ are shown in Fig.1. It shows the detected output for various setting of the threshold values by keeping $\alpha$ constant as 0.1 . The setting of $\alpha$ and $\mathrm{T}$ as 0.1 and 0.5 respectively, gives more accurate detection result for all sequences compare to other setting. The performance parameters such as False Positive (FP, no of background 
pixels that were marked as foreground), False Negative(FN, number of foreground pixels that were missed), True Positive (TP, correct detection of foreground pixels) and True Negative (TN, correct detection of background pixels) are evaluated for each sequence by comparing foreground masks with ground truth. Another parameters like Precision $(\mathrm{P})$ and Recall $(\mathrm{R})$ in which Precision reflects the false detection rate and Recall reflects the accuracy of detection results. To get robust and accurate detection result, the value of these two parameters must be as large as possible. These parameters are evaluated using following formulas.

In order to systematically and quantitatively estimate various detection algorithm, it is useful to recognize the following two important quality measures such as Recall and Precision. Precision reflect the false alarm and recall gives accuracy of detection result.

Precision $(\%)=\frac{T P}{T P+F P} * 100$

$\operatorname{Recall}(\%)=\frac{T P}{T P+F N} * 100$

We calculate Recall and Precision for various video sequences of Wallflower database. Then experimental result shows that by making different combinations of parameters like learning rate $\alpha$ and threshold value $\mathrm{T}$, we can evaluate the errors like false positive and false negative. The false negative goes on increasing by selecting higher threshold value. By increasing the value of learning rate $\alpha$, true positive increases and false positive reduced. Thus by selecting $\alpha$ as 0.1 instead of 0.01 and $\mathrm{T}$ as 0.5 instead 0.7 and 0.9 gives more accurate results compare to other combinations of $\alpha$ and $\mathrm{T}$. Appropriate setting of $\alpha$ and $\mathrm{T}$ among 25 settings are decided based on lowest value of total error for each setting. Higher values of precision and recall gives more accurate detection results. We kept the parameter values of our method same for all test sequences. However better result could be obtained by customizing the values for each sequence. The results are shown in Fig. 1

\begin{tabular}{|c|c|c|c|c|}
\hline Video & Sequence & $(0.1,0.5)$ & $(0.1,0.7)$ & $(0.1,0.9)$ \\
\hline MO & & & & \\
\hline WT & & & & \\
\hline $\mathrm{C}$ & & & & \\
\hline B & & & & \\
\hline FA & & & & \\
\hline
\end{tabular}

Fig 1: Detection result of our method for different values $\alpha$ and $T$ parameters setting for Wallflower database
As shown in Table 2. the performance evaluation parameters are calculated using detected output of our algorithm for a particular frame of video sequences whose ground truth is available. . The proposed algorithm minimize the interference of surrounding impact and remove shadow region.

Table 2. Quantitative analysis of detection results

\begin{tabular}{|c|c|c|c|c|c|c|}
\hline Video & TP & TN & FP & FN & P(\%) & R(\%) \\
\hline MO & 0 & 19200 & 0 & 0 & 100 & 100 \\
\hline WT & 12558 & 766 & 306 & 4570 & 98 & 80.34 \\
\hline C & 3399 & 1030 & 770 & 8231 & 81.53 & 83.68 \\
\hline B & 2546 & 15927 & 295 & 432 & 89.61 & 85.49 \\
\hline FA & 3754 & 13718 & 745 & 983 & 83.44 & 84.60 \\
\hline
\end{tabular}

\subsection{Object tracking using Kalman filter}

In this section, we performed several experiments to prove the feasibility of the proposed tracking method. The experimental results are shown for various conditions in indoor and outdoor environment with multiple moving objects. The results demonstrate a high performance of the proposed algorithm in the cluttered outdoor scene. The image size of all test sequences is $320 \times 240$ pixels. These experiments are implemented on the MATLAB $14 \mathrm{~b}$ platform with Intel core I5, $2.7 \mathrm{GHz}$ and 4GB memory PC. The experimental results shows that the proposed method is efficient, effective and near real time. All the objects are tracked properly in spite of the merge that occur due to occlusion.

In order to test the proposed tracking method, we use test sequence from PETS 2009 database (ftp://pets.rdg.ac.uk) [17]. This test sequence contain multiple moving objects crossing each other. Our system count the number of objects enter into the scene. It also identify the objects which are entering into the scene and leaving from the scene. We notice that some objects are not correctly identified in some frames of the sequences. Even though during the severe occlusion, objects are not separately tracked.

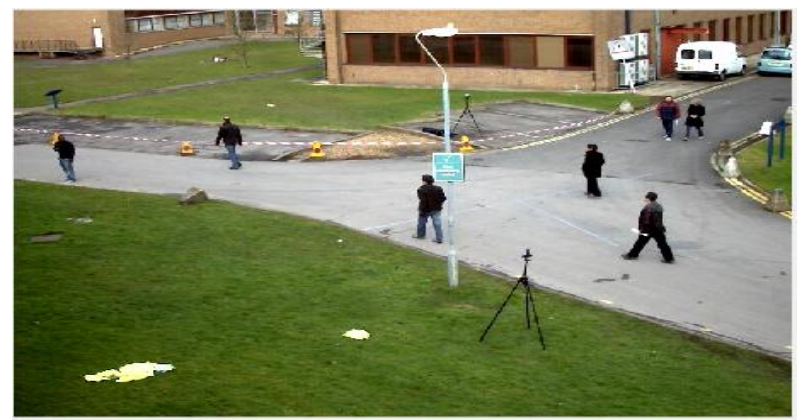

(a)

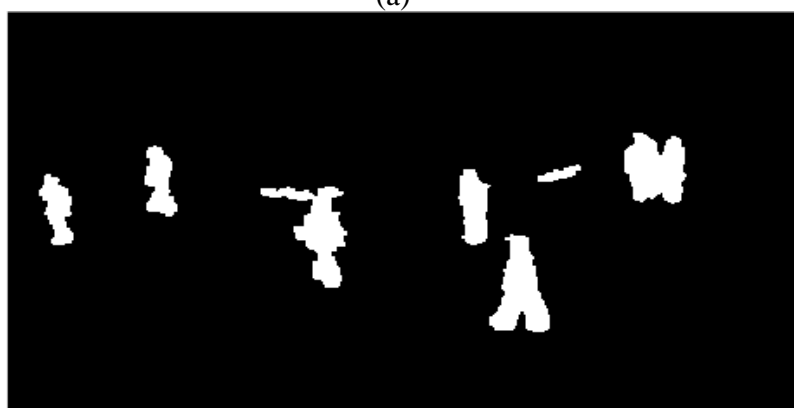

(b) 


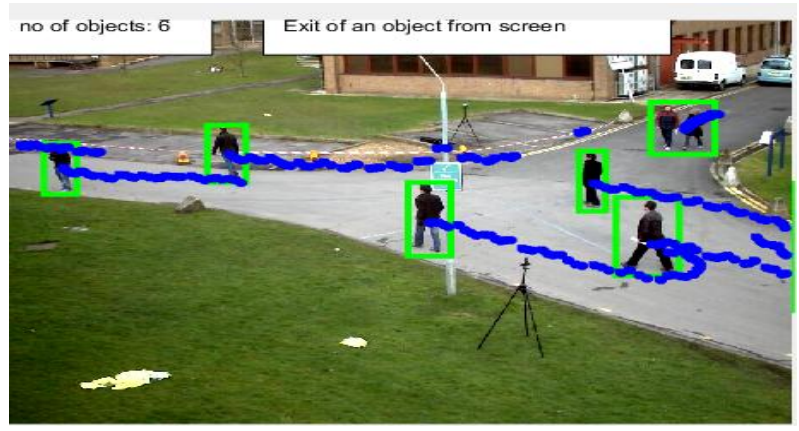

(c)

Fig 2: (a) Video frame from PET 2009 dataset (b)Detection result (c) Tracking result

\section{CONCLUSION}

We have implemented object detection based on adaptive Gaussian Mixture Model technique and object tracking using Kalman filter. It has been evaluated against several video sequences including both indoor and outdoor scenes. Gaussian Mixture Model is applied to extract foreground object from the background. Experimental results shown that GMM algorithm is more robust and accurate to detect the moving object from the complex and non-stationary background scenes compare to adaptive median filter background modeling technique. GMM can easily adapt to illumination change scene and preserve the complete edge information of moving object. There may also be the concern that the linear motion estimation may fail for objects moving in complicated nonlinear way. If the movements of objects are slow than the deviation from estimated position between successive frames then correct tracking is achieved. If mistracking is occurred for some frames by the reasons of occlusion, appearing and disappearing of the objects then the proposed Kalman tracking algorithm could recover correct track after a couple of frames.

\section{REFERENCES}

[1] Jianpeng Zhou and Jack Hoang, "Real Time Robust Human Detection and Tracking System", IEEE Trans. Pattern Analysis and Machine Intelligence, Vol.19, 2006, pp. 780-785.

[2] Grimson Wel, Stauffer C. Romano R. Lee L."Using adaptive tracking to classify and monitor activities in a site", in Proceedings.1998 IEEE Computer Society Conference on Computer Vision and Pattern Recognition (Cat. No.98CB36231). IEEE Comput.Soc. 1998.

[3] Stauffer C, Grimson W. E. L. Adaptive background mixture models for real-time tracking. in Proceedings. 1999 IEEE Computer Society Conference on Computer Vision and Pattern Recognition (Cat. No PR00149). IEEE Comput. Soc. Part Vol. 2, 1999.

[4] D. Hari Hara Santosh, P. Venkatesh,"Tracking Multiple Moving Objects Using Gaussian Mixture Model', International Journal of Soft Computing and Engineering (IJSCE), ISSN: 2231-2307, Volume-3, Issue-2, May 2013.
[5] P. Kaew TraKulPong and R. Bowden, "An Improved Adaptive Background Mixture Model for Real-Time Tracking with Shadow Detection," Proc.European Workshop Advanced Video Based Surveillance Systems, 2001.

[6] Z. Zivkovic, "Improved Adaptive Gaussian Mixture Model for Background Subtraction," Proc. Int'l Conf. Pattern Recognition, vol. 2, pp. 28-31, 2004.

[7] K. Toyama, J. Krumm, B. Brumitt, and B. Meyers, "Wallflower: Principles and Practice of Background Maintenance," Proc. IEEE Computer Vision, vol. 1, pp. 255-261, 1999.

[8] Marko Heikkila and Matti Pietika, Senior Member, IEEE, " A Texture-Based Method for Modeling the Background and Detecting Moving Objects", IEEE transactions on pattern analysis and machine intelligence, Vol. 28, No. 4, pp.657-662, April 2006.

[9] Q. Zang and R. Klette, "Robust Background Subtraction and Maintenance," Proc. Int'l Conf. Pattern Recognition, vol. 2, pp. 90- 93, 2004.

[10] A. Elgammal, R. Duraiswami, D. Harwood, and L.S. Davis, "Background and Foreground Modeling Using Nonparametric Kernel Density Estimation for Visual Surveillance," Proc. IEEE, vol. 90, no. 7, pp. 1151-1163, 2002.

[11] K. Kim, T.H. Chalidabhongse, D. Harwood, and L. Davis, "Background Modeling and Subtraction by Codebook Construction,” Proc. IEEE Int'l Conf. Image Processing, vol. 5, pp. 3061-3064, 2004

[12] J. Kato, T. Watanabe, S. Joga, J. Rittscher, and A. Blake, "An HMM-Based Segmentation Method for Traffic Monitoring Movies," IEEE Trans. Pattern Analysis and Machine Intelligence, vol. 24, no. 9, pp. 1291-1296, Sept. 2002.

[13] A. Monnet, A. Mittal, N. Paragios, and R. Visvanathan, "Background Modeling and Subtraction of Dynamic Scenes," Proc. IEEE Int'l Conf. Computer Vision, vol. 2, pp. 1305-1312, 2003.

[14] Xin Li, Kejun Wang,Wei Wang and Yang Li, "A Multiple Object Tracking Method Using Kalman Filter", Proceedings of the 2010 IEEE International Conference on Information and Automation June 20 - 23, Harbin, China.

[15] M. Mason and Z. Duric, "Using Histograms to Detect and Track Objects in Color Video," Proc. Applied Imagery Pattern Recognition Workshop, pp. 154-159, 2001

[16] Amir Gahremani, Amir Mousavinia,"Visual object tracking using Kalman filter, mean shift algorithm and spatiotemporal oriented energy features" fourth IEEE international conference on computer and knowledge engineering, 2014.

[17] Xin li, Kejun Wang," A multiple object tracking method using Kalman filter", IEEE international conference on information and automation, July 2010. 